\title{
ISWC 2012 best papers
}

\author{
Mark T. Smith • Daniel Ashbrook
}

Published online: 23 February 2013

(c) Springer-Verlag London 2013

This special issue of Pervasive and Ubiquitous Computing includes three invited papers that are extended versions of best paper-nominated submissions to the sixteenth International Symposium on Wearable Computers (ISWC), held from June 18-22, 2012, in Newcastle, UK. The first paper, entitled Airwriting: A Wearable Handwriting Recognition System by Christoph Amma, Marcus Georgi, and Tanja Schultz describes a system for the recognition of handwriting gestures in real time that allows users to write in free space in an equivalent manner to writing on a white board or wall. It includes a description of a wireless gloveworn device to sense writing movement along with a description and evaluation of the algorithms and language model used to predict handwriting. It will be of interest to researchers wanting to exploit gesturing in user interfaces by extending them to natural handwriting.

The second paper, entitled The Distant N-Gram Topic Model: A Probabilistic Approach to Mining Sequences by Katayoun Farrahi and Daniel Gatica-Perez, describes a method and mathematical model for extracting or discovering data patterns from large data sets, for example those resulting from collecting sensor data over an extended period of time. This work will be of interest to those wishing to model activity or other behavioral patterns that can occur over long-measurement intervals under relatively unstructured conditions.

M. T. Smith (ه)

Stockholm, Sweden

e-mail: msmith@kth.se

D. Ashbrook

San Jose, CA, USA

e-mail: dan@danielashbrook.com
The third paper, entitled Activity Recognition for Creatures of Habit: Energy-Efficient Embedded Classification using Prediction by Dawud Gordon, Jürgen Czerny, and Michael Beigl, was the best paper winner and describes a novel algorithm for classifying human activities reported by wearable sensors followed by a prediction of future actions. This algorithm can be used by applications to assist hardware power management, for example by activating sensors that are the most likely to be needed, while shutting off ones that are less likely to be used resulting in significant energy savings.

ISWC is overseen by the IEEE Technical Committee on Wearable Information Systems (TC WIS). ISWC has been the leading forum for wearable computing since the conference was first held in 1997. For the last 2 years, the conference has been co-located with Pervasive, uniting the growing ubiquitous computing community. Through ISWC, related conferences such as ISMAR, and special issues of journals such as this issue of PUC, TC WIS has worked to bring together the diverse range of people who are interested in wearable computing, from computing researchers to apparel designers. TC WIS concentrates on all issues related to wearing information technology, such as user interfaces, low power hardware, and design for wearability. The TC steering committee invites anyone interested in wearable information systems to join the TC. The TC website (http://www.iswc.net) has instructions on joining the TC, as well as information on upcoming events and links to previous years of ISWC. 